\title{
Complete mitochondrial genomes reveal robust phylogenetic signals and evidence of positive selection in horseshoe bats
}

\author{
Lin Zhang ${ }^{1}$, Keping Sun ${ }^{1} 2^{*}$, Gábor Csorba ${ }^{3}$, Alice Catherine Hughes ${ }^{4}$, Longru Jin ${ }^{1}$, Yanhong Xiao ${ }^{1}$ and \\ Jiang Feng ${ }^{1,5^{*}}$
}

\begin{abstract}
Background: In genus Rhinolophus, species in the Rhinolophus philippinensis and R. macrotis groups are unique because the horseshoe bats in these group have relatively low echolocation frequencies and flight speeds compared with other horseshoe bats with similar body size. The different characteristics among bat species suggest particular evolutionary processes may have occurred in this genus. To study the adaptive evidence in the mitochondrial genomes (mitogenomes) of rhinolophids, especially the mitogenomes of the species with low echolocation frequencies, we sequenced eight mitogenomes and used them for comparative studies of molecular phylogeny and adaptive evolution.
\end{abstract}

Results: Phylogenetic analysis using whole mitogenome sequences produced robust results and provided phylogenetic signals that were better than those obtained using single genes. The results supported the recent establishment of the separate macrotis group. The signals of adaptive evolution discovered in the Rhinolophus species were tested for some of the codons in two genes (ND2 and ND6) that encode NADH dehydrogenases in oxidative phosphorylation system complex I. These genes have a background of widespread purifying selection. Signals of relaxed purifying selection and positive selection were found in ND2 and ND6, respectively, based on codon models and physicochemical profiles of amino acid replacements. However, no pronounced overlap was found for non-synonymous sites in the mitogenomes of all the species with low echolocation frequencies. A signal of positive selection for ND5 was found in the branch-site model when R. philippinensis was set as the foreground branch.

Conclusions: The mitogenomes provided robust phylogenetic signals that were much more informative than the signals obtained using single mitochondrial genes. Two mitochondrial genes that encoding proteins in the oxidative phosphorylation system showed some evidence of adaptive evolution in genus Rhinolophus and the positive selection signals were tested for ND5 in R. philippinensis. These results indicate that mitochondrial protein-coding genes were targets of adaptive evolution during the evolution of Rhinolophus species, which might have contributed to a diverse range of acoustic adaptations in this genus.

Keywords: Mitogenome, Rhinolophus, Comparative analysis, Positive selection

*Correspondence: sunkp129@nenu.edu.cn; fengj@nenu.edu.cn 1 Jilin Provincial Key Laboratory of Animal Resource Conservation and Utilization, Northeast Normal University, Changchun 130117, China Full list of author information is available at the end of the article

\section{Background}

Mitochondria are the energy metabolism centers that play critical roles in adenosine triphosphate (ATP) synthesis and heat generation [1]. The oxidative phosphorylation system (OXPHOS), which is the main pathway that produces cellular energy via ATP, provides approximately 
95\% of the energy required by an organism for the basic activities of life [2-4]. There are five complexes in OXPHOS; four of these complexes (I, III, IV, and V) are co-encoded by nuclear and mitochondrial genes, whereas complex II is encoded exclusively by nuclear genes $[2,5$, $6]$. Vertebrate mitochondrial genomes (mitogenomes) comprise a large non-coding region (control region) and 37 genes; 13 encode OXPHOS proteins, two encode ribosomal RNAs (rRNAs), 22 encode transfer RNAs (tRNAs) $[7,8]$.

Mitogenomes are characterized by their small size, lack of recombination, maternal inheritance, and high substitution rates. All these properties have made mitochondrial genes ideal for used in phylogeography, species identification, and molecular phylogenetics [1, 9-12]. Mitochondrial genes are linked within the same DNA molecule, so it is expected that different mitochondrial genes share the same genealogy [9]. However, incongruent phylogenetic results have frequently been found among different mitochondrial genes, indicating these genes contain different phylogenetic signals [13-15]. Although single or a few concatenated genes have often been used to represent an entire mitogenome, the best genes or regions for a particular study are highly taxadependent $[9,16]$. Analyses of mitogenomes have proven useful for inferring recent evolutionary relationships.

Mitogenome have historically been assumed to evolve neutrally. However, the 13 mitochondrial protein-coding genes (PCGs) are expected to have experienced nonneutral changes because of their functional importance in OXPHOS $[17,18]$. Mitochondria function to supply cellular energy and produce heat and are therefore extremely sensitive to heat and energy-related selective pressure [1, 19-22]. The role of selection in the evolution of mitochondrial DNA (mtDNA) attracted little attention until recently $[3,9,23,24]$. Mitochondrial OXPHOS genes have been shown to display evidence of adaptive evolution in, for example, dogs, bats, birds and fishes [21, $22,25,26]$, and numerous studies have detected signals of positive selection in mitochondrial genes [21-23, 27], suggesting positive selection plays a strong potential role in adaptation $[28,29]$.

Bats (order Chiroptera) are unique mammal that have developed flight as their primary locomotion mode [21, 30]. Bats have undergone unique adaptive evolution for their survival and the evolution of flight is an energetically expensive adaptation. It is therefore likely that genes involved in energy metabolism would have undergone much evolutionary change. Shen et al. [21] resequenced 77 OXPHOS genes, including mitochondrial- and nuclear-encoded OXPHOS genes from four species of bats. Their results showed adaptive evolution of genes involved in energy metabolism specific on the common ancestral bat lineage [21]. Mitochondrial PCGs in particular have vital roles in energy production by directly mediating proton-pumping across the membrane [10]. Analyses of these PCGs may greatly facilitate the understanding of chiropteran evolution [30]. Complete mitogenome data are available for only 99 bat species, which is a small proportion of the $\sim$ approximately 1300 bat species [31]. Therefore, the generation and analysis of additional complete mitogenome sequences will help to further understand bat evolution.

Among the horseshoe bats (genus Rhinolophus), those in the Rhinolophus macrotis and R. philippinensis groups have lower echolocation frequencies than other rhinolophids with similar body sizes, except for $R$. osgoodi [32]. Our field observations have shown that species in these two groups have relatively low flight speeds compared with the flight speeds of other species. Evolutionary constraints on the mtDNA of birds were found to lead to the accumulation of more non-synonymous than synonymous substitutions in weakly flying and flightless birds compared with those accumulated in rapidly flying birds [22]. These unique phenomena may have a genetic and possibly adaptive basis, making the philippinensis and macrotis groups suitable for studies into the role of natural selection in determining specific traits. The macrotis group was separated from the former philippinensis group in a phylogenetic tree constructed by Zhang et al. [33] that included six closely related species; $R$. macrotis Blyth, 1844; R. siamensis Gyldenstolpe, 1917; $R$. rex Allen, 1923; $R$. osgoodi Sanborn, 1939; $R$. marshalli Thonglongya, 1973; and R. schnitzleri Wu \& Thong, 2011 (Fig. 1). The philippinensis group now includes $R$. philippinensis Waterhouse, 1843; R. montanus Goodwin, 1979; and $R$. achilles Thomas, 1900. The macrotis and philippinensis groups are widely distributed across large parts of South and Southeast Asia, are morphology conserved, and have a young radiation history [33, 34]. The initial diversification of the macrotis group began during the Pleistocene and was potentially associated with the Tibetan Plateau uplift and climate changes during that time [35]. We speculate that both these groups may have undergone distinctive evolution, which suggests the possibility that natural selection acted at the mitogenome level. Although mitogenomes have been widely used in phylogenetic studies of many mammalian groups, most molecular phylogenetic studies of horseshoe bats have been based on limited numbers of mitochondrial genes $[33,36,37]$. No mitogenome-based phylogenic study of horseshoe bats has focused on the molecular evolution of energy metabolism-related genes, and thus their evolutionary history is poorly understood.

Only two mitogenomes of bat species with low echolocation frequency relative to body size $\left(\operatorname{species}_{\text {low }}\right)$ are 


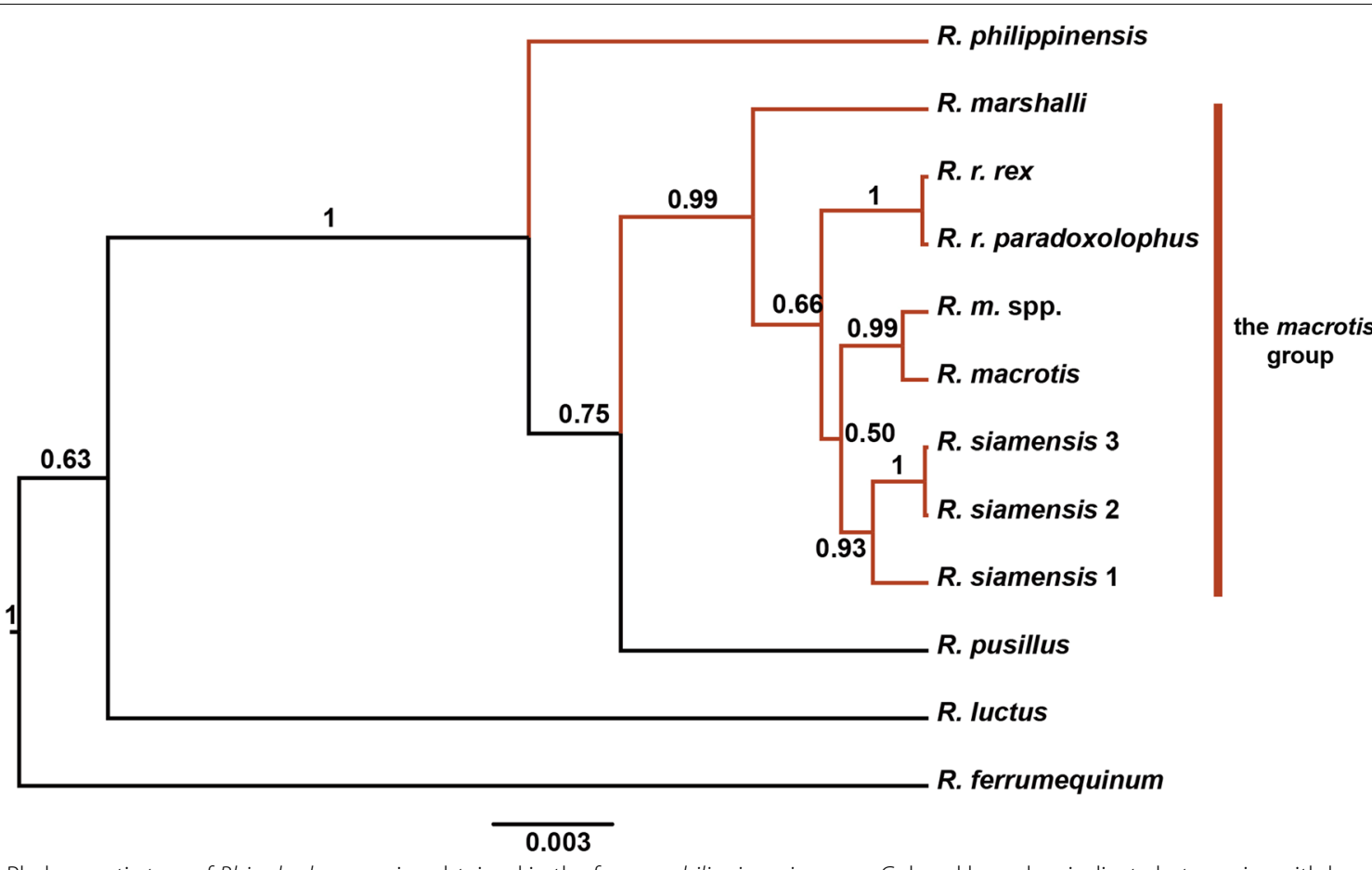

Fig. 1 Phylogenetic tree of Rhinolophus species obtained in the former philippinensis group. Colored branches indicate bat species with low echolocation frequency relative to body size. This tree is reproduced from Zhang et al. [33]

available in NCBI's GenBank: $R$. macrotis (NC_026460) and $R$. rex (NC_028536). In this study, we sequenced eight mitogenomes including two additional species in the macrotis group as well as $R$. philippinensis and $R$. pusillus Temminck, 1834. We analyzed the newly sequenced complete mitogenomes and another four published Rhinolophus mitogenomes. We performed a comparative mitogenomes analysis for species $_{\text {low }}$ to: (1) reveal characteristic and distinct nucleotide patterns; (2) reconstruct the mitochondrial phylogeny and assess the phylogenetic signals of single mitochondrial genes; and (3) detect signals of the adaptive evolution of the mitochondrial genes in genus Rhinolophus, especially those

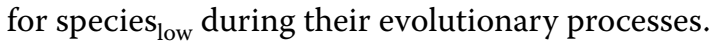

\section{Material and methods}

\section{Sampling and DNA extraction}

Tissue samples were collected from eight individuals representing six species, then DNA was extracted and sequenced (detailed sampling information is provided in Additional file 1). Of the six species, three were $R$. siamensis considering that $R$. siamensis 2 and $R$. siamensis 3 were formerly recognized as $R$. huananus and $R$. cf. siamensis, respectively, and were considered as junior synonyms of $R$. siamensis [33] (Additional file 1). Three other species $_{\text {low }}(R$. schnitzleri, $R$. achilles and R. montanus) in the macrotis and philippinensis groups were not included in this study because of their limited distribution and sampling difficulty.

All samples were initially preserved in $100 \%$ ethanol in the field, then transferred and stored at $-80{ }^{\circ} \mathrm{C}$ until DNA extraction. Tissues were collected from muscle of voucher specimens or the wing membrane (biopsy punches: 3-mm diameter) of living bats. Total genomic DNA was extracted using a UNIQ-10 column animal genomic DNA isolation kit (Sangon Biotech, Shanghai, China), following the manufacturer's instructions. To compare the patterns of mitogenome sequence evolution of the six species $_{\text {low }}$, four rhinolophid mitogenome sequences from previously published studies were downloaded from NCBI (https://www.ncbi.nlm.nih.gov/), two of which are from the macrotis group (Additional file 2).

\section{PCR amplification and sequencing}

We aligned the sequence of the 15 available complete rhinolophids mitogenomes from NCBI and the primers were designed in the conserved regions. The whole mitogenome of each sequenced individual was amplified in overlapping fragments with tens or hundreds of bases using 13 specific primers designed in Primer 5.0 [38] and Primer 6.0 [39]. The mitogenomes of R. monoceros Andersen, 1905 (NC_005433) and R. macrotis were used as reference sequences for primer design. We also designed specific primers for some regions that were not 
amplified using the 13 primers. The primer sequences used in this study are listed in Additional file 3. The PCRs and sequencing were conducted as described previously [33].

\section{Sequence annotation and bioinformatics analysis}

Sequences were aligned and assembled using Geneious 9.0 [40]. The PCGs, rRNA and tRNA genes, and noncoding regions of each mitogenome were annotated using the complete mitogenome of $R$. macrotis as the reference sequence. The 13 PCGs were translated into amino acid sequences using the vertebrate mitochondrial genetic code and checked for unexpected frameshift errors or stop codons in MEGA v.7 [41]. Nucleotide composition was calculated, and total GC content and codon usage were analyzed. Strand asymmetry was calculated as follows: AT-skew $=[\mathrm{A}-\mathrm{T}] /[\mathrm{A}+\mathrm{T}]$ and $\mathrm{GC}$ skew $=[G-C] /[G+C][42]$. DNA polymorphisms were calculated for each single gene using DnaSP v5.0 [43]. All the newly sequenced complete mitogenome sequences have been deposited in NCBI under Accession numbers MK987176-MK987183 as shown in Additional file 2.

\section{Phylogenetic analysis}

Twelve mitogenome sequences, excluding intergenic and control regions, were used in the phylogenetic analyses (Additional file 2). The PCGs, rRNAs and
tRNAs were aligned separately, and the 22 tRNAs were combined using SequenceMatrix [44] as one sequence because of the short sequence length for each tRNA. Then, all sites of 13 PCGs, 2 rRNAs and 22 tRNAs were combined as a concatenated dataset that contained 15,487 nucleotides. We used jModelTest 0.1 [45] to determine the best-fit nucleotide substitution models (Table 1) for the 13 PCGs, 12S rRNA, 16S rRNA, and the combined 22 tRNAs under the Akaike information criterion.

The phylogeny analysis was conducted using Bayesian inference with MrBayes 3.2.2 [46] and the concatenated dataset. To evaluate the phylogenetic signal for each single mitochondrial gene, single mitochondrial gene datasets with each of the 13 PCGs, 12 S and 16 S rRNAs, and the combined 22 tRNAs were used to reconstruct phylogenies. The Markov chain Monte Carlo technique was used to conduct two independent runs with four chains (two heated and two cold) simultaneously for 10 million generations, sampling every 1000 generations. After discarding the first $25 \%$ of the samples as burn-in, posterior probabilities were calculated in a consensus tree. Stationarity was assumed when the average standard deviation of split frequencies fell below 0.01 . The resulting consensus tree was visualized in Figtree. We compared tree topologies obtained from

Table 1 Best substitution models for each of 13 protein-coding genes, and comparison of phylogenetic topologies recovered by different mitochondrial datasets

\begin{tabular}{|c|c|c|c|c|}
\hline Gene & Model & The macrotis group & The macrotis complex & $\begin{array}{l}\text { R.r.rex }+ \text { R.r. } \\
\text { paradoxolophus }\end{array}$ \\
\hline ATP6 & $\operatorname{TrN}+\mathrm{G}$ & $\mathrm{N}$ & Y & Y \\
\hline ATP8 & $\mathrm{TIM} 3+1+\mathrm{G}$ & N & N & Y \\
\hline Cytb & TPM2uf $+G$ & Y & Y & Y \\
\hline $\operatorname{COX} 1$ & TPM2uf $+I+G$ & N & N & Y \\
\hline $\operatorname{cox} 2$ & $H K Y+G$ & N & N & Y \\
\hline $\operatorname{cox} 3$ & $\mathrm{TIM} 2+\mathrm{I}$ & Y & N & Y \\
\hline ND1 & $\mathrm{TIM} 2+\mathrm{G}$ & N & $\mathrm{N}$ & Y \\
\hline ND2 & $\operatorname{TrN}+\mathrm{I}+\mathrm{G}$ & $\mathrm{N}$ & $\mathrm{N}$ & Y \\
\hline ND3 & $\operatorname{TrN}+1$ & N & N & Y \\
\hline ND4 & $\operatorname{TrN}+1$ & Y & Y & Y \\
\hline ND4L & TPM2uf + I & N & N & Y \\
\hline ND5 & $\mathrm{TIM} 1+\mathrm{G}$ & Y & Y & Y \\
\hline ND6 & $\mathrm{TIM} 3+\mathrm{G}$ & Y & Y & Y \\
\hline 12SrRNA & $\mathrm{TPM} 2 \mathrm{RF}+\mathrm{I}+\mathrm{G}$ & N & $\mathrm{N}$ & Y \\
\hline $16 S$ rRNA & $\mathrm{TIM} 2+\mathrm{G}$ & $\mathrm{N}$ & N & Y \\
\hline 22 tRNAs & $H K Y+G$ & N & N & Y \\
\hline Concatenated dataset & & Y & Y & Y \\
\hline
\end{tabular}

Concatenated dataset: 13 protein-coding genes, 2 ribosomal RNA genes, and 22 transfer RNA genes. Three mitochondrial clades inferred from the mitochondrial concatenated dataset were defined

Y, phylogeny is supported; $N$, phylogeny is not supported 
each of the 16 single-gene datasets with the topology from the concatenated dataset.

\section{Analysis of selection pressure Neutral evolution}

We calculated the total number of amino acid replacements, the number of fixed non-synonymous substitutions and the number of synonymous changes for each gene using DnaSP.

According to methods used in Jacobsen et al. [65], all 13 PCGs were used to assess neutral evolution in species low $_{\text {la }}$ by calculating the linear regression between: (1) number of mutations and length in bases, (2) number of synonymous mutations and length in bases, and (3) number of non-synonymous changes and synonymous changes. The total number of amino acid replacements for each gene was corrected for gene length by calculating the number of amino acid replacements divided by the total number of amino acids in the gene.

\section{Selection analysis}

The selection level against non-synonymous substitutions relative to synonymous substitutions was measured as the ratio of the number of non-synonymous substitutions per non-synonymous sites $(\mathrm{Ka})$ to the number of synonymous substitutions per synonymous sites (Ks). To compare the differences in sequence evolution pattern between species ${ }_{\text {low }}$ and other rhinolophids, the Ka/ Ks distributions in pairwise comparisons between DNA sequences were calculated [47] for each of the 13 PCGs using $\mathrm{Ka} / \mathrm{Ks}$ calculator [48]. We used SPSS 19 [49] to assess the significance of the $\mathrm{Ka} / \mathrm{Ks}$ values.

Codon substitution has been widely used to detect adaptive signatures affecting protein evolution [50]. To identify signals of positive and purifying selection in the 13 PCGs for species $_{\text {low }}$, three codon-based selection methods were applied: HYPHY, implemented in the Datamonkey server (http://www.datamonkey.org/) [51], CodeML in PAML v4.8 [52], and TreeSAAP version 3.2 [53]. Because each method is prone to false positives, we used multiple methods with different underlying assumptions and checked for congruence [10]. A species tree (Fig. 1) from Zhang et al. [33] was used in the selection analyses.

For the selection analyses of the 13 PCGs, stop codons were removed and the ND6 sequence was reverse-complemented because $N D 6$ is on the light strand. Overlapping regions (7 bp of ATP8/ATP6 and 4 bp of ND4L/ND4) were included in the alignment twice to enable analyses of all the codons.

Codon-based selection pressure $(\omega)$ ratio Codons putatively under selection were detected using HYPHY. The
FUBAR (Fast Unbiased Bayesian AppRoximation) [54] was used to detect codons under pervasive purifying or diversifying selection and MEME (Mixed Effects Model of Evolution) [55] was used to identify pervasive and episodic positive selection. The best-fit substitution model for each dataset was selected in HYPHY [56]. Significance was assessed by posterior probability $(\mathrm{PP})>0.9$ (FUBAR) or $P$ value $<0.05$ (MEME).

CodeML, implemented in PAML, was used to run site and branch-site models by comparing a series of maximum-likelihood models to test for signatures of positive selection associated with particular codons or lineages. First, we used the free-ratio model, which allows $\omega$ to vary in different lineages. Then, we used the two-ratio model, which allows two sets of lineages to have different $\omega$ values, and used M0 (one $\omega$ ratio) to compare the lineages for foreground branches (branches for species $_{\text {low }}$ ) with background branches (all remaining branches).

The site model allowed $\omega$ to vary among codons and assume one $\omega$ ratio for all branches. Six different site models were run using equal codon frequencies as proposed by Yang et al. [57]: M0 (one $\omega$ ratio), M1a (nearly neutral), M2a (positive selection), M3 (discrete), M7 (beta), and M8 (beta and $\omega$ ). A likelihood ratio test (LRT) was used to compare a null model against a model that allowed codons to be $\omega>1$ as follows: M0 vs. M1a tested for a single $\omega$ ratio across codons, M0 vs. M3 assessed whether variable selection pressure was present among sites, and M1a vs. M2a and M7 vs. M8 tested presence of positive selection. When the LRT indicated that models predicted positive selection, a Bayes empirical Bayes (BEB) method implemented in the CodeML program of PAML was used to calculate the PPs of the codons that tested a $\omega>1$ in M2a and M8. PPs $>0.85$ were considered as supported under the BEB method [57].

The CodeML branch-site model was also run to test whether some sites had undergone positive selection in a foreground branch of the phylogenetic tree. Signals of positive selection for codons on given branches were detected by considering the lineages of each species $_{\text {low }}$ and the macrotis group as foreground branches. The alternative (MA, positive selection: $0<\omega_{0}<1, \omega_{1}=1$, $\omega_{2} \geq 1$ ) and null model (MA0, neutral evolution with $\omega_{2}=1$ fixed) were used to detect selective pressure on each branch. In all cases, an LRT was used for the pairwise model comparison and to determine which model better fits the data $(P<0.05)$. Positive selection was inferred when $\omega>1$ and the LRT was significant $(P<0.05)$. The BEB method was used to calculate PPs for site classes to determine which codon positions had experienced positive selections $(\omega>1)$ on the foreground lineages [58]. 
Radical changes in physicochemical properties of amino acids TreeSAAP was used to infer the direction of amino acid replacements by comparing mitogenomes codon-bycodon under the predefined phylogeny. TreeSAAP assigns weight values to non-synonymous codon changes based on the effects of amino-acid replacements on 20 physicochemical properties using the evolutionary tree (accuracy of detecting selection $>85 \%$ ) [59]. We tested significant deviation from neutral evolution by calculating a $\mathrm{z}$-score, and positive selection was assigned when the number of inferred amino acid replacements significantly exceeded the number expected by chance alone, yielding positive $\mathrm{z}$-scores. The overall amino acid replacements are scored from 1 to 8 , with eight as the most significant change. The analysis was run using a window size of 20 codons and sliding in 1-codon increment. To minimize false positives, only amino acid replacements with strong effects on protein biochemistry ( $\mathrm{z}$-score $>3.09$ (corresponding to $P<0.001$ ) and magnitude categories $>6$ ) were considered to potentially be evolving under positive selection [60].

\section{Results}

\section{Characteristics of the mitogenome}

The complete mitogenomes sequenced in this study shared similar sequence characteristics. Each mitogenome was a closed-circular DNA molecule that contained a typical set of 37 genes: 13 PCGs, 22 tRNAs, 2 rRNAs, and a non-coding region. The gene arrangement, organization, and content of the mitogenomes were identical to those of other Chiroptera mitogenomes. The mitogenome ranged in size from $16,811 \mathrm{bp}$ in $R$. rex paradoxolophus to $16,897 \mathrm{bp}$ in $R$. $m$. spp. (sensu Tu et al. [61]) (Additional file 4). The length variation across different species was relatively low in the coding genes and the differences in length resulted largely from variations in the lengths of non-coding regions, especially in the control region. Except for ND6 and eight of the tRNA genes, which were on the light strand, the other mitochondrial genes (PCGs, rRNAs, and other tRNAs) and the control region were on the heavy strand.

All these rhinolophid mitogenomes showed highly similar nucleotide composition biases (Additional file 2). All the mitogenomes were AT rich, with the AT content ranging from $56.2 \%$ (R. siamensis 3 ) to $57.1 \%$ (R. philippinensis). The AT content of the species low $_{\text {mitogenomes }}$ was slightly lower than that of the other rhinolophids mitogenomes. All these mitogenomes were highly congruent in base skewness, with a positive AT-skew (0.099 to 0.111$)$ and a markedly negative GC-skew $(-0.351$ to -0.321 ), suggesting strand heterogeneity in nucleotide composition.

\section{Mitochondrial phylogenetic analysis Whole mitogenomic data}

The molecular phylogeny reconstructed based on the concatenated dataset yielded topological relationships with high PPs (PP $>0.95$ was considered as strong support in Bayesian inference) (Fig. 2). The topology supported the polyphyly for the former philippinensis group already noted by Zhang et al. [33]. Except for $R$. philippinensis, the other species in the former philippinensis group represented a monophyletic clade with high nodal support, named as the macrotis group in a previous study [33]. The macrotis group consisted of two sister clades: (1) the macrotis complex with robust statistical support and (2) $R$. marshalli $+R$. rex with a relatively low PP of 0.64 .

\section{Single mitochondrial genes}

The alignment length and parsimony informative sites for each of the 13 mitochondrial PCGs are provided in Additional file 4. All phylogenies reconstructed based on 16 single-gene datasets showed different topologies (Additional file 5), and none of these topologies was identical to the phylogeny obtained with the concatenated dataset. Varied tree topologies among the different datasets indicated incongruent phylogenetic signals across the PCGs. The percentage of polymorphic sites varied among the 16 datasets, ranging from $10.51 \%$ in tRNAs to $31.65 \%$ in ND4L (Additional file 4). We compared the single-gene trees with the tree for the concatenated dataset in three clades (the macrotis group, the macrotis complex and $R$. rex, including the subspecies $R . r$. paradoxolophus) (Table 1). Six genes (ATP6, Cytb, COX3, ND4L, ND5 and ND6) supported at least one of the three clades recovered by the concatenated dataset, whereas Cytb, ND4L, ND5 and ND6 supported all three clades.

\section{Analysis of selective pressure Testing for neutral evolution}

The total number of mutations and the number of synonymous changes both showed significant linear relationships with gene length. The number of non-synonymous changes was correlated with the number of synonymous changes, although the correlation was not significant. Compared with the other genes, ND2 and ND5 showed relative high proportions of non-synonymous changes (Fig. 3).

\section{Distribution of amino acid replacements across protein domains}

The proportion of amino acid replacements, which was scaled by gene length, ranged from 0.001 (COX1) to 0.034 (ATP8) across 13 PCGs in species low (Additional file 4). The proportions of amino acid replacements that were 


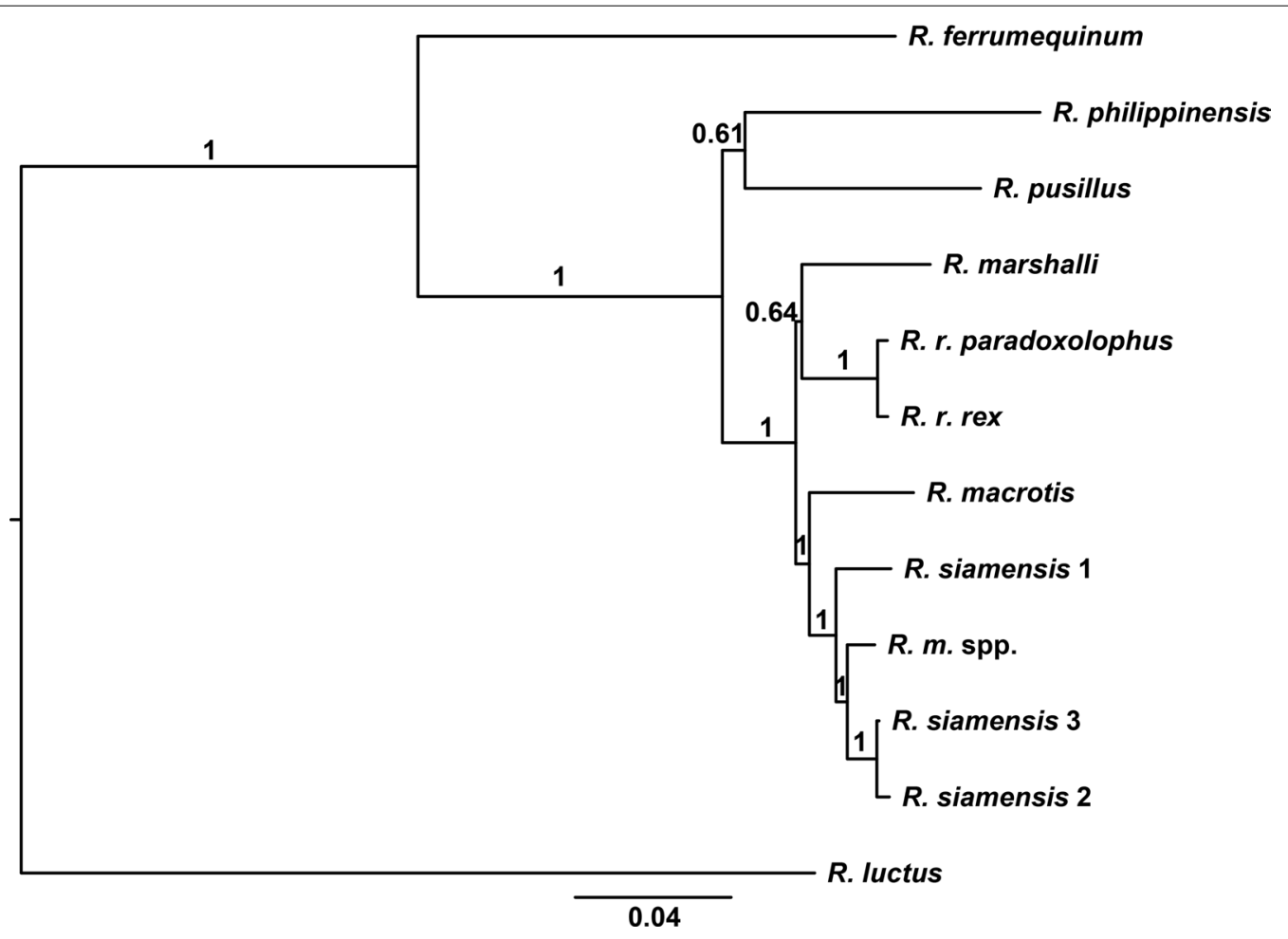

Fig. 2 Phylogenetic tree of Rhinolophus species reconstructed using the concatenated dataset. The concatenated dataset contained the whole mitogenome sequences excluding the control region. The tree was constructed using Bayesian inference analyses in MrBayes. Posterior probabilities values are shown on the nodes

fixed for the $\mathrm{NADH}$ dehydrogenase genes ranged from 0.0101 (ND4) to 0.0246 (ND6), and that for ATP8 (0.034) was higher than it was for the other genes (Fig. 4); these genes belong to OXPHOS complex I and complex V, respectively. Thus, complex I and complex V harbored a higher proportion of amino acid replacements than the other OXPHOS complexes.

\section{Evidence of natural selection across all 13 mitochondrial protein-coding genes}

Selective pressure on different species clusters The species $_{\text {low }}$ have lower echolocation frequencies than other species with similar body sizes (Fig. 5). Three genes (ATP6, ND2, and ND5) were found to have significantly different $\mathrm{Ka} / \mathrm{Ks}$ values $(P=0.002,0.018,0.002$, respectively) between the species ${ }_{\text {low }}$ and other species clusters (Table 2), indicating that the mitochondrial PCGs of the species in these two clusters experienced different evolutionary pressures.

The one-ratio model analyses showed that the $\omega$ values for the 13 PCGs were significantly $<1$, suggesting that these Rhinolophus genes had experienced constrained selective pressure to maintain their functions.

The site model comparisons indicated that the mitogenome has evolved in a non-neutral pattern in
Rhinolophus species. The LRT values for M0 vs. M3 were highly significant for eight genes (ATP6, COX3, Cytb, ND1, ND2, ND3, ND4, and ND6, $P<0.05)$ and M0 vs. M1a showed that eight genes had evolved non-neutrally (COX3, Cytb, ND1, ND2, ND3, ND4, ND5, and ND6, $P<0.05)$. These results indicate that the selection pressure $(\omega)$ for these genes varied among sites. No sites evolved under positive selection as indicated by M1a vs. M2a (LRT not significant). Two codons in ND6 were under positive selection as identified by M7 vs. M8, and for one codon $\left(N D 6_{101 \mathrm{~T}}\right)$ the PP $(\mathrm{PP}=0.99)$ was higher than the $\mathrm{PP}=0.85$ threshold according to the $\mathrm{BEB}$ analysis (Additional file 6, Table 3). The different codon numbers between the model comparisons are expected because M2a was a highly conservative test with lower power than M8 [23, 62, 63].

The branch-site model was tested for each species $_{\text {low }}$ and the macrotis group, and the results showed positive selection in four sites of ND5 when $R$. philippinensis was set as the foreground branch, and the PP was high for only one site $\left(N D 5_{543 S}, \mathrm{PP}=0.992\right)$ (Additional file 6, Table 3). The MA vs. Ma0 and MA vs. M1a comparisons were significant for $N D 5_{543 S}$. These results imply that this codon position in $R$. philippinensis experienced positive 

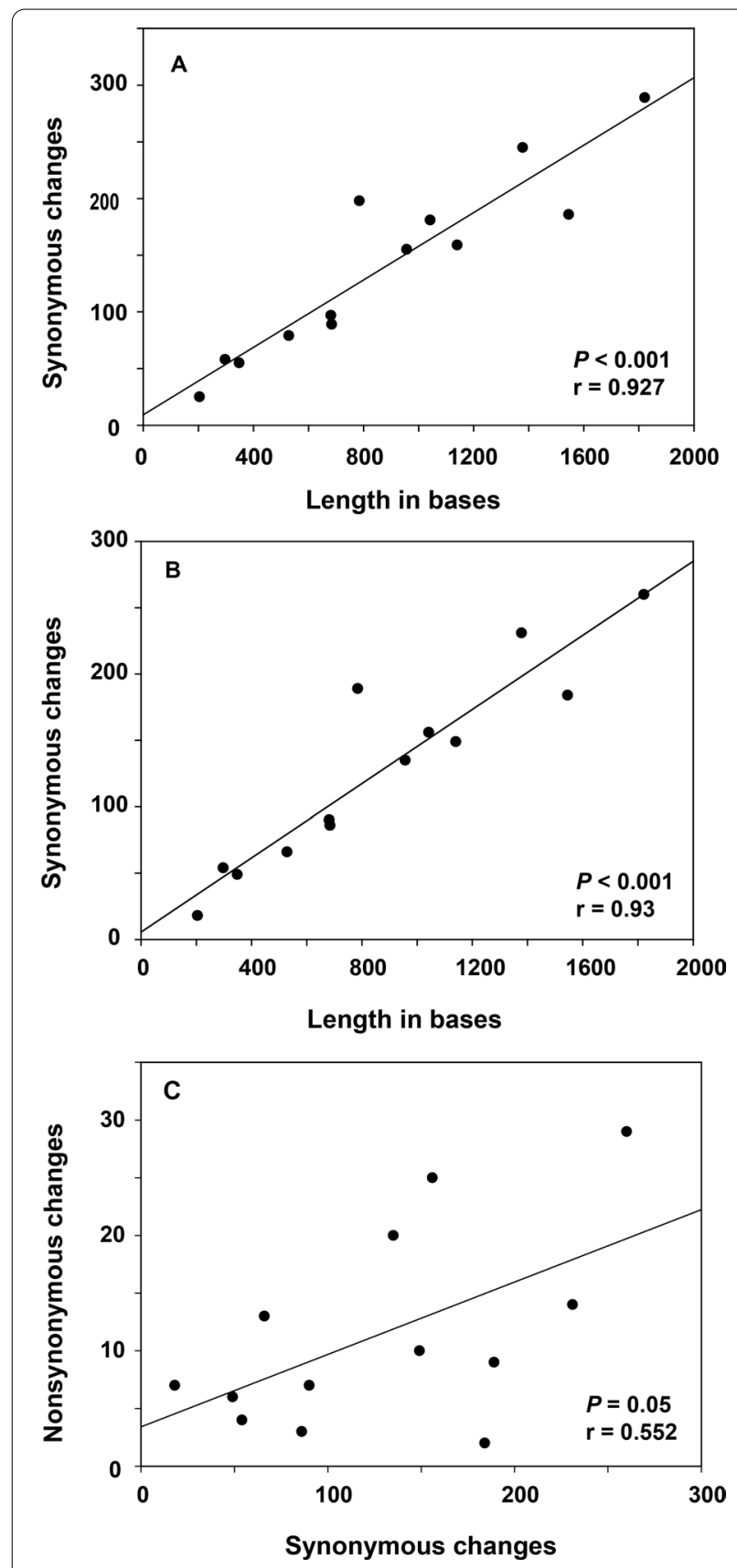

Fig. 3 Correlation analyses. The 13 mitochondrial protein-coding genes of the bat species with low echolocation frequency relative to body size are indicated by dots. The lines are the best-fitted line. A Correlation between total number of mutations and gene length. B Correlation between synonymous mutations and gene length. C Correlation between non-synonymous and synonymous mutations. The correlation coefficient $r$ and $P$ value are shown in the lower right corner for linear regression.

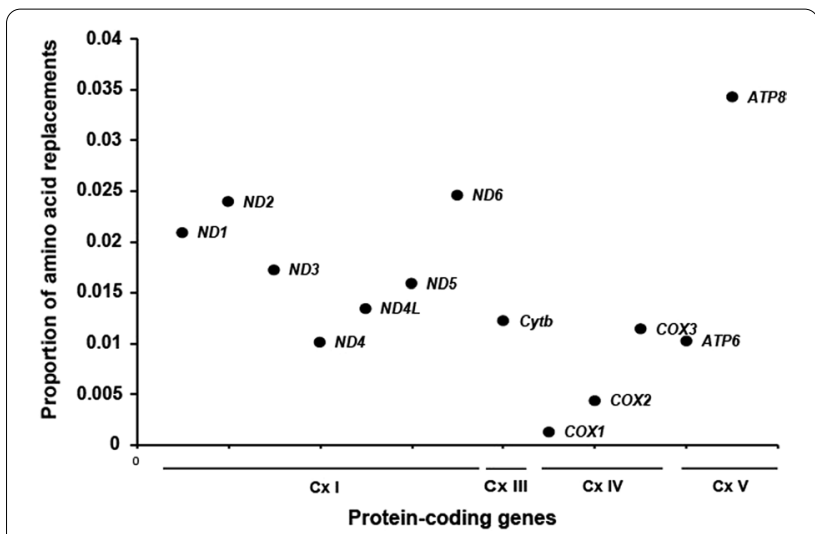

Fig. 4 Fixed amino acid replacements in bat species with low echolocation frequency relative to body size. The proportion of amino acid replacements was calculated as the total number of replacements in each protein-coding gene divided by the gene length. The dots indicate the 13 different mitochondrial OXPHOS genes and the lines below the $x$-axis indicate the genes in the OXPHOS complexes (Cx)

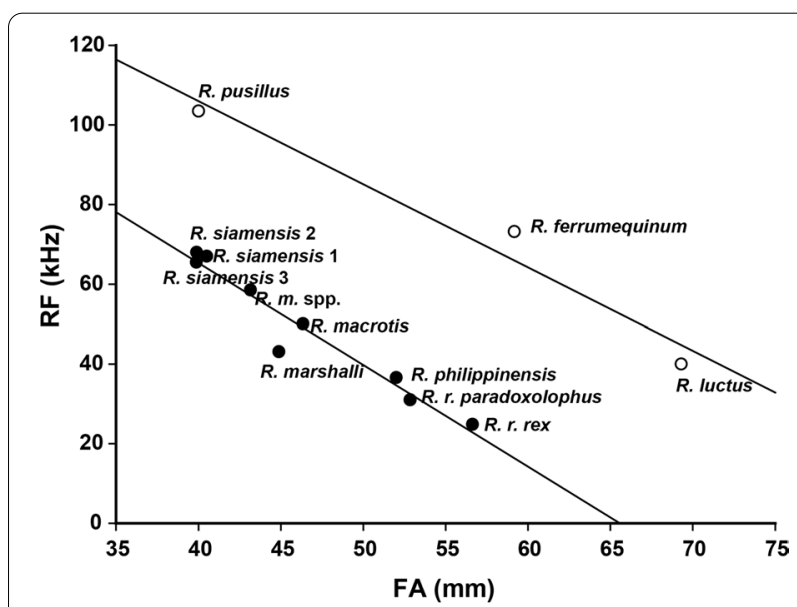

Fig. 5 Relationship between average forearm length (FA) and resting frequency (RF) for the Rhinolophus species. Solid circles indicate species with low echolocation frequency relative to body size; empty circles indicate the other species

selection and accumulated a high number of non-synonymous mutations.

The results from HYPHY showed codons spread across all 13 PCGs with evidence of pervasive purifying selection within Rhinolophus. No codons involved in diversifying positive selection were detected by FUBAR, which suggested pervasive purifying selection occurred across all mitochondrial PCGs. MEME 
indicated two codons showed evidence of episodic positive selection in ND2 and ND5, and these codons were also found in the site model $\left(N D 2_{331 \text { I }}\right.$, not significant) and branch-site model $\left(N D 5_{5435}\right)$ implemented in PAML. The REL analysis identified one positively selected site in ND6 $\left(N D 6_{101 \mathrm{~T}}\right.$, Bayes factor $=981.166$, $P=0.98$ ). Thus, PAML and HYPHY together suggested two amino acid replacements as candidates for positive selection (Table 3), and both sites supported by the two independent tests were in OXPHOS complex I. Another candidate site $\left(N D 2_{3311}\right)$ which was significant in the MEME test, was also located in complex I.

Evolution of amino-acid physicochemical properties inferred by TreeSAAP The results from TreeSAAP for the 13 PCGs of Rhinolophus suggested a prevalence of purifying selection and several significant physicochemical changes, which suggested positive selection. This result was similar to the result obtained with the

Table 2 Non-synonymous/synonymous (Ka/Ks) values for 13 protein-coding genes of the species in two clusters

\begin{tabular}{|c|c|c|c|}
\hline Gene & $\mathrm{ka} / \mathrm{ks}_{\text {low }}{ }^{\mathrm{a}}$ & $\mathrm{ka} / \mathrm{ks}_{\text {normal }} \mathrm{b}$ & $P$ value ${ }^{c}$ \\
\hline ATP6 & 1.047779 & 0.012706 & $0.002^{* *}$ \\
\hline ATP8 & 0.104115 & 0.080252 & 1 \\
\hline $\operatorname{cox} 1$ & 0.002006 & 0.00317 & 0.136 \\
\hline $\operatorname{cox} 2$ & 0.01567 & 0.013083 & 0.433 \\
\hline $\operatorname{cox} 3$ & 0.017378 & 0.017603 & 0.941 \\
\hline Cytb & 0.024837 & 0.024236 & 0.501 \\
\hline ND1 & 0.038619 & 0.029385 & 0.823 \\
\hline ND2 & 0.050525 & 0.078366 & $0.018^{*}$ \\
\hline ND3 & 0.031403 & 0.032856 & 0.14 \\
\hline ND4 & 0.024325 & 0.016991 & 0.228 \\
\hline ND4L & 0.031965 & 0.054756 & 0.092 \\
\hline ND5 & 0.036546 & 0.049335 & $0.002^{* *}$ \\
\hline ND6 & 0.066561 & 0.08568 & 0.096 \\
\hline
\end{tabular}

${ }^{\mathrm{a}} \mathrm{Ka} / \mathrm{Ks}_{\text {low' }}$ mean $\mathrm{Ka} / \mathrm{Ks}$ values for the species that have lower echolocation frequencies than other species with similar body sizes

${ }^{\mathrm{b}} \mathrm{Ka} / \mathrm{Ks}_{\text {normal, }}$ mean $\mathrm{Ka} / \mathrm{Ks}$ for the species in the other cluster

'Values in bold font indicate significant differences. Note: ${ }^{*} 0.01<P<0.05$, ${ }^{* *}$ $0.001<P<0.01$

codon models. We found that 19 of the 20 physicochemical properties were under purifying selection (global negative $\mathrm{z}$-score $<-3.09, P<0.001$, Additional file 7). Evidence for positive selection was found for two physicochemical properties, with global z-scores above the significance threshold $(\mathrm{z}>3.09, P<0.001)$. The property equilibrium constant (ionization of $\mathrm{COOH}$ ), which scored category level $8(\mathrm{z}=27.4)$, and solvent accessible reduction ratio, which scored category level $7(\mathrm{z}=5.3)$, indicated radical amino acid replacements had occurred by positive selection.

The identified sites $N D 2_{331 \mathrm{I}}$ and $N D 6_{101 \mathrm{~T}}$ were considered as possible loci of positive selection, which scored category level 8 and significant positive z-scores ( $\mathrm{z}>3.09, P<0.001)$ only in radical changes suggested for the equilibrium constant (ionization of $\mathrm{COOH}$ ). Notably, different methods found signatures of positive selection along with amino acid property changes in similar regions $\left(N D 2_{3311}\right.$ and $\left.N D 6_{101 T}\right)$ (Fig. 6). The replacement fixed within $N D 5\left(N D 5_{5435}\right)$ was supported as potentially under positive selection for buriedness $(\mathrm{z}>3.09, P<0.01)$ in TreeSAAP, but this was not significant.

\section{Discussion}

In this comprehensive study, we present a comparative analysis of the mitogenomes of Rhinolophus species and examined natural selection at the molecular genetic

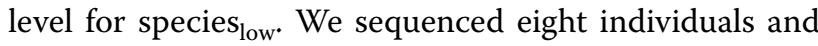
included 12 mitogenomes in the analysis. The results showed that several codons and lineages were under positive selection.

\section{Characters of the mitogenomes of species}

Mammalian mitogenomes are typically $16 \mathrm{~kb}$ in size [30] and the mitogenome reported have similar sizes. The general features of the mitogenomes, including genome size, gene content, gene arrangement, codon usage, and secondary structures of the tRNAs, were highly conversed in species $_{\text {low }}$, and similar to those of other $R h i$ nolophus species. Analysis of variation in structure, nucleotide composition, codon bias, and other parameters among bats will improve our understanding of the evolutionary processes involved in their diversity. Therefore, we investigated the diversity in eight new bat mitogenomes to identify features associated with their evolution.

The mitogenome of $R . \mathrm{m}$. spp. was found to be longer and the AT content of the mitogenomes of all the species in the macrotis group was lower than those of the other investigated Rhinolophus species, including R. philippinensis, a representative of a closely related species group. Because AT pairs have two hydrogen bonds and GC pairs have three hydrogen bonds, low AT content is considered more stable than high AT content, and therefore high AT content may lead to a less stable structure and possibly accelerated evolutionary adaptation. The lower AT content for the macrotis group may be an important adaptive 
Table 3 Positive selected sites tested using different methods

\begin{tabular}{|c|c|c|c|c|}
\hline Gene & Codon & Method & Parameter & Significant \\
\hline \multirow[t]{3}{*}{ ND2 } & 331 & Codeml (site model) & $\begin{array}{l}\omega 2=52.46859(\mathrm{M} 2 \mathrm{a}) \\
\omega=2.37570(\mathrm{M} 8)\end{array}$ & $\begin{array}{l}\mathrm{PP}=0.671(\mathrm{M} 2 \mathrm{a}) \\
\mathrm{PP}=0.809(\mathrm{M} 8)\end{array}$ \\
\hline & 331 & FEL & $\omega>1, L R T=3.52$ & $P=0.06$ \\
\hline & 331 & MEME & $\omega>1, \mathrm{LRT}=4.22$ & $P=0.045^{*}$ \\
\hline \multirow[t]{2}{*}{ ND5 } & 543 & Codeml (branch-site model) & $\omega 2 b=40.98885$ & $P P=0.992^{* *}$ \\
\hline & 543 & MEME & $\omega>1, \mathrm{LRT}=14.45$ & $P=0.0003^{* * *}$ \\
\hline \multirow[t]{2}{*}{ ND6 } & 101 & Codeml (site model) & $\begin{array}{l}\omega 2=6.32703(\mathrm{M} 2 \mathrm{a}) \\
\omega=6.63018(\mathrm{M} 8)\end{array}$ & $\begin{array}{l}\mathrm{PP}=0.925^{*}(\mathrm{M} 2 \mathrm{a}) \\
\mathrm{PP}=0.986^{*}(\mathrm{M} 8)\end{array}$ \\
\hline & 101 & REL & Bayes factor $=981.166$ & $P P=0.98^{*}$ \\
\hline
\end{tabular}

* indicate sites under positive selection. Note: ${ }^{*} 0.01<\mathrm{P}<0.05$ or $\mathrm{PP}>0.95,{ }^{* *} \mathrm{PP}>0.99,{ }^{* * *} \mathrm{P}<0.001$

characteristic, reflecting mitochondrial DNA variations and evolutionary adaptation.

\section{Phylogenetic analyses for species}

Mitochondrial genes have been widely used in studies of phylogeny, phylogeography, and evolution $[1,9,10$, $17,23,63]$. Phylogenies obtained from complete mitogenomes represent the highest phylogenetic branch supports and therefore clearest taxonomic resolution [9]. Our phylogenetic analyses based on a concatenated dataset recovered a highly supported topology that was congruent with previous comprehensive phylogenetic analyses using a concatenated dataset of four mitochondrial genes for the former philippinensis group [33]. In this study, the polyphyly for the former philippinensis group and monophyly for the newly established macrotis group were well supported. The species in the macrotis group showed highly similar morphological characteristics, and clustered in a highly supported monophyletic clade, which was similar to the relationships obtained from the concatenated mitochondrial dataset in Zhang et al. [33]. Rhinolophus $m$. spp. clustered with $R$. siamensis, which was also found by Tu et al. [61] and Zhang et al. [33]. These findings combined with those of previous studies suggest that ancient introgression may exist because of climate fluctuations when bats diversified, and ancestral polymorphism was preserved because of the female philopatry and male-biased dispersal $[33,61]$. $R$. r. rex and R. r. paradoxolophus clustered in one clade, which verified the species delimitation that $R . r$. paradoxolophus is a subspecies of $R$. r. rex as reported previously [32,33]. $R$. marshalli clustered with $R$. rex, which is inconsistent with the results of the molecular study based on a concatenated multilocus dataset in Zhang et al. [33] but in accordance with the anatomical traits including the nasal foliation and cranial morphology [34].

Because of their linked nature, single mitochondrial genes are expected to carry the same phylogenetic signal as the whole mitogenome [9]. However, our results showed incongruent phylogenetic topologies among different genes and a different percentage of polymorphic sites in a single gene from different species, which implied the incongruent information was included in these genes. These findings may result from the varying evolutionary and selection pressure, as reported for other species $[9,13,15]$.

Although recombination does not normally occur in mitogenomes [4], different regions have different evolutionary rates $[9,64-66]$, and we also observed this in the phylogenetic analyses. Several studies have indicated that phylogenies obtained from mitogenomes are better than those obtained from single genes, and the most informative mitochondrial genes that generated phylogenies similar to the whole mitogenome have been identified $[9,13$, 67]. In this study, none of the single genes recovered the same topology as the concatenated dataset. However, we identified four genes (Cytb, ND4L, ND5, and ND6) that produced topologies similar to the concatenated mitogenome dataset in three defined clades.

Selection that causes differences in non-synonymous rates among mitochondrial genes is also important because it produces variable evolutionary rates that result from differences in the strength of purifying selection caused by functional constraints $[66,68]$. Although the numbers of neutrally evolving synonymous mutations are highly correlated with the lengths of the corresponding genes (Fig. 3B), reflecting neutral evolution, the relative rates of non-synonymous mutations differ between genes (Fig. 3C). This is consistent with gene-specific differences in purifying selection.

\section{Analyses of selective pressures in the mitochondrial genes} We explored signatures of natural selection in the Rhinolophus mitogenomes. We detected a strong and widespread signature of $\omega<1$ across the 13 PCGs, which is evidence of purifying selection on these genes that have important functions in OXPHOS. Purifying selection appears to be the main evolutionary force that affects 


\section{ND2}

\begin{tabular}{|c|c|}
\hline R. macrotis & LTYTTSLTMF PSTNNMKIKWQF \\
\hline R. m. spp. & LTYTTSLTMFPSTNNS \\
\hline R. r. paradoxolophus & LTYTTSLTMFPSTNNMKIKW \\
\hline R.r. rex & LTYTTSLTMF PSTNNMKI. \\
\hline R. siamensis 1 & LTYTTSLTMF PSTNNMKIK \\
\hline R. siamensis 2 & LTYTTSLTMFPSTNNMKIKW \\
\hline R. siamensis 3 & LTYTTSLTMFPSTNNMKIK \\
\hline R. marshalli & LTYSTS LTMF PSTNNMKII \\
\hline R. philippinensis & LTYTTSLTMFPSTNNMKIK \\
\hline R. pusillus & LTYTTSLTMI \\
\hline R. ferrumequinum & LTYTTSLTMFPSTNNMKIKV \\
\hline R. luctus & ГYTTSLTMF PSTNNMK I KV \\
\hline
\end{tabular}

\section{ND6}

R. macrotis
$R$. m. spp.
R. r. paradoxolophus
R. r. rex
R. siamensis 1
R. siamensis 2
R. siamensis 3
R. marshalli
R. philippinensis
R. pusillus
R. ferrumequinum
R. luctus

101

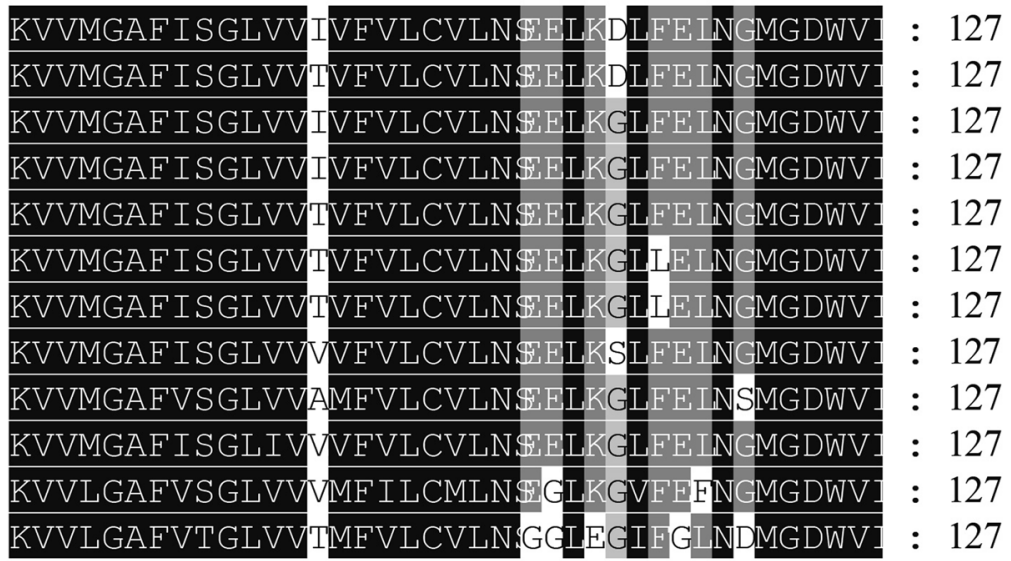

Fig. 6 Multi-alignments of partial amino acid sequences encoded by ND2 and ND6 for the Rhinolophus species. Sites ND2 $2_{3311}$ and ND6 1017 are marked

mitogenome evolution in bats. This result is consistent with the results of studies in other species such as fishes and mammals $[3,19]$. Although the mitogenomes evolved under purifying selection to conserve the functionality of OXPHOS proteins $[4,68]$, we also found heterogeneous rates of $\omega$ across sites, and evidence of positive selection $(\omega>1)$ in some codons. Positive selection in mitogenomes has been studied in a wide range of taxa, and has been associated with adaptation to extreme environments, size, and migration behaviour $[9,21,22$, 69]. Mitochondrial genetic variation was shown to affect ATP synthesis, mitochondrial content, and even the electrochemical gradient [70], suggesting the strongly potential role of mitogenomes in adaptation $[18,71]$.

The NADH dehydrogenase complex is the first and largest enzyme complex in the oxidative phosphorylation pathway in the mitochondrial electron transport chain $[3,4]$. Previous studies on vertebrate species found positive selection signals in genes that encode proteins of OXPHOS complex I, including ND2, ND5, and ND6 $[3,18,23,72,73]$. We found signatures of positive selection in Rhinolophus species according to the site model in CodeML and codon tests in HYPHY (Table 3). $N D 6_{101 \mathrm{~T}}$ was found to have sites undergoing positive selection and changes in physicochemical properties occurred in the similar equilibrium constant (ionization of $\mathrm{COOH}$ ) property for $N D 2_{3311}$ and $N D 6_{101 \mathrm{~T}}$. The significant changes in the equilibrium constant (ionization of $\mathrm{COOH}$ ) suggested adaptation to changing metabolic requirements has occurred [17]. Most physicochemical changes occur in regions genes that are likely under less conservative evolutionary constraints [65]. Genes that encode the $\mathrm{NADH}$ dehydrogenase subunits were less informative [9], and some of these genes were longer and/or had high 
percentages of polymorphic sites than other genes (Additional file 4), and therefore may be more likely to cause property changes. Our results are concordant with these findings.

ND2 shows several patterns compatible with positive selection. ND2 harbors a high percentage of the total amino acid changes and has relatively more non-synonymous substitutions than other genes in Rhinolophus mitogenomes (Fig. 3B). Moreover, the codon-based tests for positive selection in PAML (site model) and HYPHY (FEL) found little evidence of positive selection for $N D 2_{3311}$. Although $\omega>1$ was tested in the positive selection models, it was not significant in the model comparisons. However, MEME in HYPHY showed significance for codons $N D 2_{323}(P=0.01)$ and $N D 2_{331}(P=0.045)$, implying episodic diversifying selection occurred. The TreeSAAP results showed significant changes of in the equilibrium constant (ionization of $\mathrm{COOH}$ ) property for $N D 2_{3311}$. Thus, we conclude that ND2 was most likely under relaxed purifying selection. Jacobsen et al. [65] also proposed that relaxed purifying selection was driving the evolution of ND2 by affecting mostly regions, included position 331, that have lower functional relevance. They also concluded codon 331 in ND2 was under possible diversifying positive selection [65].

We did not find shared non-synonymous changes in all species $_{\text {low }}$ (Fig. 6), but the Ka/Ks values of three genes were significantly different between species ${ }_{\text {low }}$ and the other species. In particular, ATP6 (Table 2) had significantly higher $\mathrm{Ka} / \mathrm{Ks}$ values in species ${ }_{\text {low }}$ than it had in the other species (Table 2), suggesting possible connections between codon changes and phenotype differences. For other flying animals such as insects and birds, adaptive evolution and more non-synonymous nucleotide substitutions accumulated in the mitochondrial genes of weakly locomotive animals $[20,22]$. For species $_{\text {low }}$ with relatively low flight speeds, their lower dispersal ability may result in lower effective population sizes, leading to a higher probability of reaching fixation for most nonsynonymous mutations [22, 74]. However, more evidence is needed to validate this conjecture.

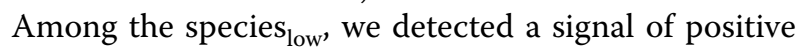
selection in $R$. philippinensis. $N D 5_{5435}$ was identified as a significant amino acid variation when the clade of $R$. philippinensis was set as the foreground lineage under the branch-site model in PAML. $R$. philippinensis is a young species with a distribution range across the Southeast Asian tropics [33, 34], and it is distributed allopatrically with other species low $_{\text {. Variation in mitochondrial }}$ OXPHOS genes may convey a signal of adaptation to the environment, particularly climatic conditions [63, 75-77]. Thus, ecological environment adaptation may lead $R$. philippinensis to be energetically different and undergoing natural selection that is different from species of the macrotis group. Further analyses and additional insights into the molecular adaptation of mitochondrial DNA in response to the tropical habitats of R. philippinensis are needed.

\section{Conclusion}

Mitogenome studies have increased over the years, but, analyses of complete bat mitogenomes are still very rare. We obtained eight new complete mitogenomes representing species of the macrotis group, $R$. philippinensis, and $R$. pusillus to evaluate the molecular phylogeny and adaptive evolution. The known mitogenomes of the Rhinolophus genus vary in length from $16,775 \mathrm{bp}$ (R. luctus) to $16,897 \mathrm{bp}(R$. m. spp.) and encode 37 genes, which have conserved general features. The phylogenetic topology reconstructed from the concatenated dataset corroborated the recently recognized macrotis group. The phylogenetic analyses results suggested the concatenated dataset performed better than single genes. Among the single genes, Cytb, ND4, ND5, and ND6 were the most informative in the phylogenetic analyses. Selective pressure analysis demonstrated that complex I and complex $\mathrm{V}$ were under relatively strong purifying selection, whereas, for complex IV, purifying selection was relatively weak. Purifying selection is widespread in mitogenomes and it plays a major role in maintaining OXPHOS function during mitogenome evolution. We found evidence of positive selection in genes that encode proteins in complex I. $N D 6$ was shown to be under positive selection and ND2 was inferred from codon tests to experience relaxed purifying selection in Rhinolophus species. ND5 was shown to be under positive selection in $R$. philippinensis, suggesting ecological environment adaptation had occurred. Further work is needed to discover the molecular adaptation mechanism of mitochondrial DNA in the Rhinolophus genus.

\section{Abbreviations}

Mitogenome: Mitochondrial genome; OXPHOS: Oxidative phosphorylation system; PCG: Protein-coding gene; ATP: Adenosine triphosphate; rRNA: Ribosomal RNA; PP: Posterior probability; FUBAR: Fast Unbiased Bayesian AppRoximation; MEME: Mixed Effects Model of Evolution; LRT: Likelihood ratio test; BEB: Bayes empirical bayes.

\section{Supplementary Information}

The online version contains supplementary material available at https://doi. org/10.1186/s12862-021-01926-2.

Additional file 1: Table S1. Voucher numbers of samples used in this study.

Additional file 2: Table S2. Length and base composition of each genomic component for species used in this study.

Additional file 3: Table S3. PCR primers used in this study. 
Additional file 4: Table S4. Polymorphic sites of 13 PCGs, 2 rRNA genes and the combined 22 tRNA genes for species in this study, and the muta-

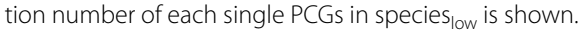

Additional file 5: Figure S1. Phylogenetic trees reconstructed from each single gene in MrBayes and posterior probability values are shown on the nodes.

Additional file 6: Table S5. Results of positive selection based on site model and branch-site model testing in protein-coding mitochondrial genes.

Additional file 7: Table S6. Significant values $(P<0.001)$ of TreeSAAP positive and negative $z$-score for amino acid physiochemical properties among radical categories 6,7 and 8 .

\section{Acknowledgements}

We thank Margaret Biswas, Ph.D, from Liwen Bianji (Edanz) (www.liwenbianji. $\mathrm{cn} /$ ) for editing the English text of a draft of this manuscript. And, we are grateful to Roniel Epilar for editorial input, and two anonymous reviewers for their constructive comments, which helped us substantially improve the manuscript.

\section{Authors' contributions}

LZ, KPS, JF, LRJ conceived and designed the study. GG and ACH offered the samples. LZ performed the experiments and analyzed the data. LZ, KPS and YHX modified the manuscript. All authors read and approved the final manuscript.

\section{Funding}

This work was supported by the National Natural Science Foundation of China [Grant numbers 31770403, 32171525, 31961123001 and 32171481], the Fundamental Research Funds for the Central Universities (2412021 QD021), and the Program for Introducing Talents to Universities (B16011). GC was supported by the Northeast Normal University, Changchun, and by the Hungarian Scientific Research Fund (OTKA) [Grant number K112440].

\section{Availability of data and materials}

The final complete mitogenome sequences sequenced in this manuscript are deposited in NCBI with Accession Number MK987176-MK987183.

\section{Declarations}

\section{Ethics approval and consent to participate}

\author{
Not applicable.
}

\section{Consent for publication}

Not applicable.

\section{Competing interests}

The authors declare no competing interests.

\section{Author details}

${ }^{1}$ Jilin Provincial Key Laboratory of Animal Resource Conservation and Utilization, Northeast Normal University, Changchun 130117, China. ${ }^{2}$ Key Laboratory of Vegetation Ecology, Ministry of Education, Changchun, China. ${ }^{3}$ Department of Zoology, Hungarian Natural History Museum, Budapest, Hungary. ${ }^{4}$ Centre for Integrative Conservation, Xishuangbanna Tropical Botanical Garden, Chinese Academy of Sciences, Menglun, Mengla County 666303, Yunnan, China. ${ }^{5}$ College of Life Science, Jilin Agricultural University, Changchun 130118, China.

Received: 29 April 2021 Accepted: 25 October 2021

Published online: 03 November 2021

\section{References}

1. Wang Y, Shen Y, Feng C, Zhao K, Song Z, Zhang Y, Yang L, He S. Mitogenomic perspectives on the origin of Tibetan loaches and their adaptation to high altitude. Sci Rep. 2016;6:29690.

2. Saraste M. Oxidative phosphorylation at the fin de siècle. Science. 1999;283(5407):1488-93.

3. Fonseca RRD, Johnson WE, O'Brien SJ, Ramos MJ, Antunes A. The adaptive evolution of the mammalian mitochondrial genome. BMC Genomics. 2008;9(1):119.

4. Ballard JWO, Whitlock MC. The incomplete natural history of mitochondria. Mol Ecol. 2010;13(4):729-44.

5. Rand DM, Haney RA, Fry AJ. Cytonuclear coevolution: the genomics of cooperation. Trends Ecol Evol. 2004;19(12):645-53.

6. Scheffler IE. Molecular genetics of succinate. Quinone oxidoreductase in eukaryotes. Prog Nucleic Acid Res Mol Biol. 1998;60:267-315.

7. Boore JL. Animal mitochondrial genomes. Nucleic Acids Res. 1999;27(8):1767-80.

8. Wolstenholme DR. Animal mitochondrial DNA: structure and evolution. Int Rev Cytol. 1992;141(6):173.

9. Yuan ML, Zhang QL, Zhang L, Jia CL, Li XP, Yang XZ, Feng RQ. Mitochondrial phylogeny, divergence history and high-altitude adaptation of grassland caterpillars (Lepidoptera: Lymantriinae: Gynaephora) inhabiting the Tibetan Plateau. Mol Phylogenet Evol. 2018;122:116-24.

10. Lamb AM, Gan HM, Greening C, Joseph L, Lee YP, Moran-Ordonez A, Sunnucks P, Pavlova A. Climate-driven mitochondrial selection: a test in Australian songbirds. Mol Ecol. 2018;27(4):898-918.

11. Toews D, Brelsford A. The biogeography of mitochondrial and nuclear discordance in animals. Mol Ecol. 2012;21(16):3907-30.

12. Esquerré $D$, Donnellan S, Brennan IG, Lemmon AR, Keogh JS. Phylogenomics, biogeography, and morphometrics reveal rapid phenotypic evolution in pythons after crossing wallace's line. Syst Biol. 2020;69(2):1039-51.

13. Havird JC, Santos SR. Performance of single and concatenated sets of mitochondrial genes at inferring metazoan relationships relative to full mitogenome data. PLoS ONE. 2014;9(1):e84080.

14. Meiklejohn KA, Danielson MJ, Faircloth BC, Glenn TC, Braun EL, Kimball RT. Incongruence among different mitochondrial regions: a case study using complete mitogenomes. Mol Phylogenet Evol. 2014;78:314-23.

15. Wang J, Zhang L, Zhang QL, Zhou M, Wang X, Yang XZ, Yuan M. Comparative mitogenomic analysis of mirid bugs (Hemiptera: Miridae) and evaluation of potential DNA barcoding markers. PeerJ. 2017;5:e3661.

16. Duchêne S, Archer FI, Vilstrup JT, Caballero S, Morin PA. Mitogenome phylogenetics: the impact of using single regions and partitioning schemes on topology, substitution rate and divergence time estimation. PLoS ONE. 2011;6(11):e27138.

17. Romero PE, Weigand AM, Pfenninger M. Positive selection on panpulmonate mitogenomes provide new clues on adaptations to terrestrial life. BMC Evol Biol. 2016;16(1):164.

18. Garvin MR, Bielawski JP, Sazanov LA, Gharrett AJ. Review and meta-analysis of natural selection in mitochondrial Complex I in Metazoans. J Zool Syst Evol Res. 2015;53(1):1-17.

19. Sun Y, Shen Y, Irwin DM, Zhang Y. Evaluating the roles of energetic functional constraints on teleost mitochondrial-encoded protein evolution. Mol Biol Evol. 2011;28(1):39-44.

20. Yang $Y, X u$ S, Xu J, Guo Y, Yang G. Adaptive evolution of mitochondrial energy metabolism genes associated with increased energy demand in flying insects. PLoS ONE. 2014;9(6):e99120.

21. Shen Y, Liang L, Zhu Z, Zhou W, Irwin DM, Zhang Y. Adaptive evolution of energy metabolism genes and the origin of flight in bats. Proc Natl Acad Sci USA. 2010;107(19):8666-71.

22. Shen YY, Shi P, Sun YB, Zhang YP. Relaxation of selective constraints on avian mitochondrial DNA following the degeneration of flight ability. Genome Res. 2009;19(10):1760-5.

23. Morales HE, Pavlova A, Joseph L, Sunnucks P. Positive and purifying selection in mitochondrial genomes of a bird with mitonuclear discordance. Mol Ecol. 2015;24(11):2820-37. 
24. Tian R, Xu S, Chai S, Yin D, Zakon H, Yang G. Stronger selective constraint on downstream genes in the oxidative phosphorylation pathway of cetaceans. J Evol Biol. 2018:31(2):217-28.

25. Bjornerfeldt S, Webster MT, Vila C. Relaxation of selective constraint on dog mitochondrial DNA following domestication. Genome Res. 2006;16(8):990-4.

26. Wang $X$, Zhang $Y$, Zhang H, Qin G, Lin Q. Complete mitochondrial genomes of eight seahorses and pipefishes (Syngnathiformes: Syngnathidae): insight into the adaptive radiation of syngnathid fishes. BMC Evol Biol. 2019;19(1):119.

27. Ramos E, Freitas L, Nery MF. The role of selection in the evolution of marine turtles mitogenomes. Sci Rep. 2020;10(1):16953.

28. Sarkar I, Dey P, Sharma SK, Ray SD, Kochiganti VHS, Singh R, Pramod $P$, Singh RP. Turdoides affinis mitogenome reveals the translational efficiency and importance of NADH dehydrogenase complex-I in the Leiothrichidae family. Sci Rep. 2020;10:16202.

29. Li JY, Song ZL, Yan GY, He LS. The complete mitochondrial genome of the largest amphipod, Alicella gigantea: Insight into its phylogenetic relationships and deep sea adaptive characters. Int J Biol Macromol. 2019;141:570-7.

30. Meganathan PR, Pagan HJ, McCulloch ES, Stevens RD, Ray DA. Complete mitochondrial genome sequences of three bats species and whole genome mitochondrial analyses reveal patterns of codon bias and lend support to a basal split in Chiroptera. Gene. 2012;492(1):121-9.

31. Fenton MB, Simmons NB. Bats. A world of science and mystery. Chicago: University of Chicago Press; 2014.

32. Zhang L, Jones G, Zhang J, Zhu G, Parsons S, Rossiter SJ, Zhang S. Recent surveys of bats (Mammalia: Chiroptera) from China. I. Rhinolophidae and Hipposideridae. Acta Chiropterol. 2009;11(1):71-88.

33. Zhang L, Sun K, Liu T, Zhao H, Csorba G, Jin L, Thong VD, Feng J. Multilocus phylogeny and species delimitation within the philippinensis group (Chiroptera: Rhinolophidae). Zool Scr. 2018;47(6):655-72.

34. Csorba G, Ujhelyi P, Thomas N. Horseshoe bats of the world (Chiroptera: Rhinolophidae). Bishop's Castle: Alana Ecology Ltd; 2003.

35. Zhisheng A, Kutzbach JE, Prell WL, Porter SC. Evolution of Asian monsoons and phased uplift of the Himalaya-Tibetan plateau since Late Miocene times. Nature. 2001;411(6833):62-6.

36. Guillén-Servent A, Francis CM, Ricklefs RE. Horseshoe bats of the world (Chiroptera: Rhinolophidae): phylogeny and biogeography of the horseshoe bats. Bishop's Castle: Alana Ecology Ltd; 2003.

37. Francis CM, Borisenko AV, Ivanova NV, Eger JL, Lim BK, Guillen-Servent A, Kruskop SV, Mackie I, Hebert PD. The role of DNA barcodes in understanding and conservation of mammal diversity in southeast Asia. PLoS ONE. 2010;5(9):e12575.

38. Clarke KR, Gorley RN. Primer version 5.0: user manual/tutorial. 2001.

39. Clarke K, Gorley R. "Primer v6." User manual/tutorial, plymouth routine in multivariate ecological research. 2006

40. Kearse M, Moir R, Wilson AC, Stoneshavas S, Cheung M, Sturrock S, Buxton S, Cooper A, Markowitz S, Duran C. Geneious Basic: an integrated and extendable desktop software platform for the organization and analysis of sequence data. Bioinformatics. 2012;28(12):1647-9.

41. Kumar S, Nei M, Dudley J, Tamura K. MEGA: A biologist-centric software for evolutionary analysis of DNA and protein sequences. Brief Bioinform. 2008;9(4):299-306.

42. Perna NT, Kocher TD. Patterns of nucleotide composition at fourfold degenerate sites of animal mitochondrial genomes. J Mol Evol. 1995;41(3):353-8.

43. Librado P, Rozas J. DnaSP v5: a software for comprehensive analysis of DNA polymorphism data. Bioinformatics. 2009;25(11):1451-2.

44. Vaidya G, Lohman DJ, Meier R. SequenceMatrix: concatenation software for the fast assembly of multi-gene datasets with character set and codon information. Cladistics-Int J Willi Henning Soc. 2011;27(2):171-80.

45. Posada D. jModelTest: phylogenetic model averaging. Mol Biol Evol. 2008;25(7):1253-6.

46. Ronquist F, Teslenko M, van der Mark P, Ayres DL, Darling A, Hohna S, Larget B, Liu L, Suchard MA, Huelsenbeck JP. MrBayes 3.2: efficient Bayesian phylogenetic inference and model choice across a large model space. Syst Biol. 2012;61(3):539-42.

47. Nei M, Gojobori T. Simple methods for estimating the numbers of synonymous and nonsynonymous nucleotide substitutions. Mol Biol Evol. 1986;3(5):418-26.
48. Zhang Z, Li J, Zhao XQ, Wang J, Wong GK-S, Yu J. KaKs_Calculator: calculating $\mathrm{Ka}$ and $\mathrm{Ks}$ through model selection and model averaging. Genom Proteom Bioinform. 2006;4(4):259-63.

49. Norusis M. Spss I: IBM SPSS statistics 19 guide to data Analysis: International Edition. 2011

50. Zhai W, Nielsen R, Goldman N, Yang Z. Looking for Darwin in genomic sequences-validity and success of statistical methods. Mol Biol Evol. 2012;29(10):2889-93.

51. Pond SLK, Frost SDW. Datamonkey: rapid detection of selective pressure on individual sites of codon alignments. Bioinformatics. 2005;21(10):2531-3.

52. Yang Z. PAML 4: phylogenetic analysis by maximum likelihood. Mol Biol Evol. 2007;24(8):1586-91.

53. Steve W, Justin J, Smith MJ, Crandall KA, Mcclellan DA. TreeSAAP: selection on amino acid properties using phylogenetic trees. Bioinformatics. 2003;19(5):671-2.

54. Murrell B, Moola S, Mabona A, Weighill T, Sheward D, Pond SLK, Scheffler K. FUBAR: a fast, unconstrained bayesian AppRoximation for inferring selection. Mol Biol Evol. 2013;30(5):1196-205.

55. Murrell B, Wertheim JO, Moola S, Weighill T, Scheffler K, Pond SLK. Detecting individual sites subject to episodic diversifying selection. PLoS Genet. 2012;8(7):e1002764.

56. Delport W, Poon AFY, Frost SDW, Pond SLK. Datamonkey 2010: a suite of phylogenetic analysis tools for evolutionary biology. Bioinformatics. 2010;26(19):2455-7.

57. Yang Z, Wong WSW, Rasmus N. Bayes empirical bayes inference of amino acid sites under positive selection. Mol Biol Evol. 2005;22(4):1107-18.

58. Zhang J, Nielsen R, Yang Z. Evaluation of an improved branch-site likelihood method for detecting positive selection at the molecular level. Mol Biol Evol. 2005;22(12):2472-9.

59. Mcclellan DA, Ellison DD. Assessing and improving the accuracy of detecting protein adaptation with the TreeSAAP analytical software. Int J Bioinform Res Appl. 2010;6(2):120-33.

60. Mcclellan DA, Palfreyman EJ, Smith MJ, Moss JL, Christensen RG, Sailsbery JK. Physicochemical evolution and molecular adaptation of the cetacean and artiodactyl Cytochrome b proteins. Mol Biol Evol. 2005;22(3):437-55.

61. Tu VT, Alexandre H, Tamás G, Satoru A, Dai F, Thanh HT, Son NT, Furey NM, Csorba G. Integrative taxonomy of the Rhinolophus macrotis complex (Chiroptera, Rhinolophidae) in Vietnam and nearby regions. J Zool Syst Evol Res. 2017:55:177-98.

62. Anisimova M, Bielawski JP, Yang Z. Accuracy and power of the likelihood ratio test in detecting adaptive molecular evolution. Mol Biol Evol. 2001;18(8):1585-92.

63. Slimen HB, Awadi A, Tolesa ZG, Knauer F, Alves PC, Makni M, Suchentrunk F. Positive selection on the mitochondrial ATP synthase 6 and the NADH dehydrogenase 2 genes across 22 hare species (genus Lepus). J Zool Syst Evol Res. 2018:56(3):428-43.

64. Whitehead A. Comparative mitochondrial genomics within and among species of killifish. BMC Evol Biol. 2009;9(1):11.

65. Jacobsen MW, Fonseca RRD, Bernatchez L, Hansen MM. Comparative analysis of complete mitochondrial genomes suggests that relaxed purifying selection is driving high nonsynonymous evolutionary rate of the NADH2 gene in whitefish (Coregonus ssp.). Mol Phylogenet Evol. 2016;95:161-70.

66. Sarah D, Louis B, Blier PU. A comparative mitogenomic analysis of the potential adaptive value of Arctic charr mtDNA introgression in brook charr populations (Salvelinus fontinalis Mitchill). Mol Biol Evol. 2002;19(11):1902-9.

67. Nadimi M, Daubois L, Hijri M. Mitochondrial comparative genomics and phylogenetic signal assessment of mtDNA among arbuscular mycorrhizal fungi. Mol Phylogenet Evol. 2016;98:74-83.

68. Meiklejohn CD, Montooth KL, Rand DM. Positive and negative selection on the mitochondrial genome. Trends Genet. 2007;23(6):259-63.

69. Ballard JW, Melvin RG, Katewa SD, Maas K. Mitochondrial DNA variation is associated with measurable differences in life-history traits and mitochondrial metabolism in Drosophila simulans. Evolution. 2007;61(7):1735-47.

70. Latorre-Pellicer A, Moreno-Loshuertos R, Lechuga-Vieco AV, SánchezCabo F, Torroja C, Acín-Pérez R, Calvo E, Aix E, González-Guerra A, Logan A. Mitochondrial and nuclear DNA matching shapes metabolism and healthy ageing. Nature. 2016;535(7613):561. 
71. Pavlova A, Gan HM, Lee YP, Austin CM, Gilligan DM, Lintermans M, Sunnucks P. Purifying selection and genetic drift shaped Pleistocene evolution of the mitochondrial genome in an endangered Australian freshwater fish. Heredity. 2017;118(5):466-76.

72. Brandt U. Energy converting NADH. Quinone oxidoreductase (Complex I). Ann Rev Biochem. 2006;75(1):69-92.

73. Toews D, Mandic M, Richards JG, Irwin DE. Migration, mitochondria, and the Yellow-Rumped Warbler. Evolution. 2014;68(1):241-55.

74. Kimura M. On the probability of fixation of mutant genes in a population. Genetics. 1962;47:713-9.

75. Balloux F, Handley LJ, Jombart T, Liu H, Manica A. Climate shaped the worldwide distribution of human mitochondrial DNA sequence variation. Proc Biol Sci. 2009;276(1672):3447-55
76. Consuegra S, John E, Verspoor E, De Leaniz CG. Patterns of natural selection acting on the mitochondrial genome of a locally adapted fish species. Genet Sel Evol. 2015;47(1):58.

77. Melo-Ferreira J, Vilela J, Fonseca MM, Da FR, Boursot P, Alves PC. The elusive nature of adaptive mitochondrial DNA evolution of an arctic lineage prone to frequent introgression. Genome Biol Evol. 2014;6(4):886-96.

\section{Publisher's Note}

Springer Nature remains neutral with regard to jurisdictional claims in published maps and institutional affiliations.
Ready to submit your research? Choose BMC and benefit from:

- fast, convenient online submission

- thorough peer review by experienced researchers in your field

- rapid publication on acceptance

- support for research data, including large and complex data types

- gold Open Access which fosters wider collaboration and increased citations

- maximum visibility for your research: over 100M website views per year

At BMC, research is always in progress.

Learn more biomedcentral.com/submissions 